\title{
Quand suspecter une maladie auto-inflammatoire chez l'adulte?
}

Stéphanie Ducharme-Bénard ${ }^{1,2,3}$, Gilles Grateau ${ }^{2,3 *}$, Sophie Georgin-Lavialle ${ }^{2,3}$

${ }^{1}$ Service de médecine interne, Hôpital Sacré-Cour de Montréal, Université de Montréal, Québec, Canada. ${ }^{2}$ Service de médecine interne, Hôpital Tenon de l'AP-HP, Université Sorbonne, Paris, France. ${ }^{3}$ Centre de Référence des Maladies Auto-Inflammatoires rares et des Amyloses (CEREMAIA).

Adresse pour correspondance: Stéphanie Ducharme-Bénard : Email: stephanie.ducharme-benard@umontreal.ca

Submitted: 19 October 2019; Accepted: 14 November 2019; Published: 18 November 2020.

DOI: http://dx.doi.org/10.22374/cjgim.v15i4.391

\begin{abstract}
Résumé
Le domaine des maladies auto-inflammatoires (MAI) est en plein essor, avec la découverte de nouvelles maladies à chaque année. À ce jour, plus d'une trentaine de MAI sont recensées. Plusieurs d'entre elles débutent ou sont diagnostiquées à lâge adulte. Il est important de les reconnaitre pour mieux les traiter et ainsi prévenir les conséquences, parfois graves, reliées à l'inflammation chronique ou récidivante qu'elles génèrent.
\end{abstract}

\begin{abstract}
The field of auto-inflammatory diseases (AIDs) is booming, with new diseases being discovered every year. To date, more than 30 AIDs have been identified. Many of them begin or are diagnosed in adulthood. It is important to recognize them in order to better treat them and thus prevent the sometimes serious consequences related to the chronic or recurrent inflammation they generate.
\end{abstract}

\section{Introduction}

Les maladies auto-inflammatoires (MAI) sont définies comme étant des maladies causées par un défaut ou dérégulation du système immunitaire inné, marqué par la présence d'inflammation intermittente ou continue ainsi que par l'absence d'un rôle pathogénique primaire du système immunitaire adaptif. ${ }^{1}$ La méconnaissance de ces maladies entraîne probablement une sous-estimation de leur prévalence. Sous cette réserve, celle-ci varie considérablement selon le type de MAI et la population à l'étude, allant de 1:400 pour la fièvre méditerranéenne familiale en Turquie à moins de 1:1000 000 pour le déficit en mévalonate kinase en Europe. ${ }^{2,3}$ Bien qu'initialement considérées comme des maladies à début pédiatrique, certaines MAI peuvent débuter ou être diagnostiquées à lâge adulte du fait d'une errance diagnostique non négligeable. ${ }^{4-6}$ Par conséquent, compte tenu des manifestations cliniques hétérogènes des MAI, l'interniste peut jouer un rôle important dans leur identification et leur prise en charge.

Les objectifs principaux de cette revue sont de présenter brièvement la pathophysiologie des MAI, décrire la présentation clinique des MAI les plus fréquentes et proposer une démarche diagnostique à l'intention de l'interniste.

\section{La pathophysiologie}

L'immunité innée constitue la défense rapide et de première ligne du corps. Normalement, en présence de signaux de danger endogènes ou exogènes, les récepteurs de cellules telles que les neutrophiles et les macrophages activent une cascade moléculaire menant à la sécrétion d'une importante quantité de cytokines pro-inflammatoires. ${ }^{7}$ Une anomalie au niveau des différentes étapes de ce processus ou de ses mécanismes régulateurs peut engendrer le développement d'une MAI. À l'inverse, les maladies 


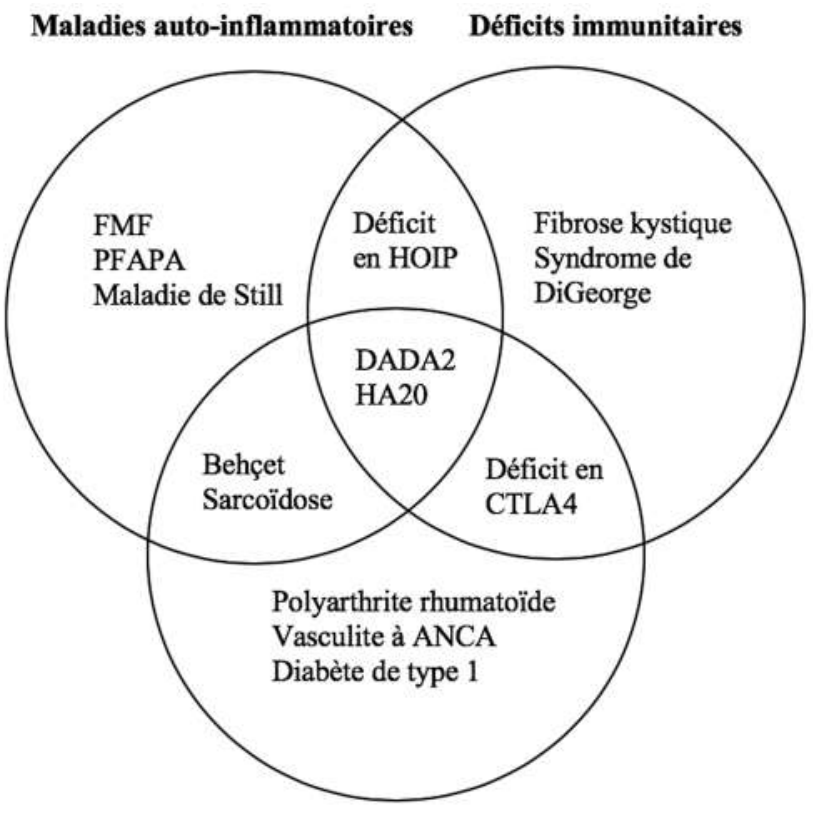

Maladies auto-immunes

Figure 1. Interaction entre les MAI, les maladies auto-immunes et les déficits immunitaires. Cette figure ne se veut pas exhaustive : les maladies représentées ont été choisies aléatoirement à titre d'exemple.

auto-immunes découlent d'une perturbation de l'immunité adaptive, entraînant la perte de tolérance des lymphocytes $\mathrm{T}$ et $\mathrm{B}$ ainsi que la production d'anticorps contre les antigènes natifs. Malgré cette dichotomie, les MAI, les maladies auto-immunes et les déficits immunitaires peuvent parfois présenter des phénotypes qui se chevauchent, selon l'hypo- et/ou l'hyper-activation du système immunitaire inné et/ou adaptif caractérisant les différentes maladies (Figure 1). ${ }^{7}$

\section{Les présentations cliniques}

Premier scénario: symptômes ayant débuté dans l'enfance

\section{A. Les 4 MAI monogéniques dites « historiques » et le syndrome de PFAPA}

La fièvre méditerranéenne familiale (FMF) est une maladie récessive associée à des mutations du gène $M E F V .{ }^{8,9}$ La population originaire du bassin méditerranéen est principalement affectée, avec plus de 100000 individus atteints. ${ }^{10}$ La FMF se manifeste par des épisodes fébriles récidivants d'une durée habituelle de 72 heures, caractérisés par une inflammation des séreuses avec au premier plan des douleurs abdominales pseudo-chirurgicales et parfois des douleurs thoraciques, articulaires et/ou testiculaires (Table 1). ${ }^{11}$ Celles-ci peuvent s'accompagner d'une éruption cutanée infrapoplitée appelée pseudoérysipèle (Figure 2). ${ }^{12}$ Sans relation temporelle avec ces symptômes, des myalgies des membres inférieurs, spontanées ou induites par l'activité physique, sont fréquentes. ${ }^{11,13}$ La FMF étant la plus amyloïdogène des MAI, la découverte d'une amylose AA peut occasionnellement être la seule manifestation clinique de la maladie. ${ }^{14,15}$ Lamylose AA est cependant plus souvent révélatrice d'une FMF typique ou paucisymptomatique non-diagnostiquée et/ou non-traitée. ${ }^{16}$

Les cryopyrinopathies ou CAPS (pour Cryopyrinassociated periodic syndrome) sont associées à des mutations dominantes du gène NLRP $3 .{ }^{17,18}$ Chez une minorité significative, des néo-mutations et des mutations somatiques sont également décrites. ${ }^{19}$ Les CAPS étaient anciennement regroupés en 3 syndromes de sévérité croissante : l'urticaire familial au froid (FCAS), le syndrome de Muckle-Wells et le syndrome chronique, infantile, neurologique, cutané, articulaire (CINCA). ${ }^{20}$ Cette nomenclature n'est plus adéquate devant le spectre clinique large de la maladie. ${ }^{1}$ Alors que l'urticaire fébrile au froid est un signe quasi-constant (Figure 3), ${ }^{21-23}$ une évolution chronique avec méningite aseptique, papilloedème et surdité neurosensorielle s'observe dans les formes les plus sévères. ${ }^{24}$

Le TRAPS (pour TNF receptor-associated periodic fever syndrome) est une maladie dominante rare associée à des mutations du gène TNFRSF1A. ${ }^{25}$ Les symptômes cardinaux constituent une fièvre prolongée d'au minimum une semaine, voire continue chez une minorité des patients, avec une douleur abdominale, des arthromyalgies souvent migratrices, et/ou une éruption cutanée classiquement située au niveau de la zone myalgique et migrant de façon centrifuge avec celle-ci. ${ }^{26}$ Présent chez une minorité de patients, l’edème périorbitaire est considéré comme un signe spécifique du TRAPS. ${ }^{26}$ Les épisodes symptomatiques s'accompagnent presque toujours d'un important syndrome inflammatoire biologique. ${ }^{27}$

Le MKD (pour Mevalonate kinase deficiency) est une maladie récessive rare due à des mutations du gène $M V K{ }^{28}$ Lors des crises, l'excès d'acide mévalonique se traduit par une acidurie mévalonique élevée; son dosage urinaire en crise constitue un test de dépistage sensible et spécifique, ${ }^{29}$ à l'inverse de la mesure du taux d'immunoglobuline (Ig) D sérique qui n’est pas indiquée. ${ }^{30}$ Les poussées fébriles débutent généralement avant l'âge de 2 ans, durent 3 à 6 jours et sont classiquement déclenchées par la vaccination. ${ }^{28}$ Elles s'accompagnent de douleur abdominale, diarrhée et/ou vomissement, aphtose buccale, adénopathie cervicale, arthromyalgies et éruption cutanée polymorphe. ${ }^{31,32}$ Les céphalées et les troubles neuropsychiatriques ne sont pas rares. ${ }^{31,33}$

Bien que d’origine polygénique, c'est-à-dire sans mutation monogénique mendélienne ou somatique identifiée, le syndrome PFAPA (pour Periodic fever, aphthous stomatitis, pharyngitis and adenitis) demeure la MAI pédiatrique la plus fréquente. ${ }^{34} \mathrm{Il}$ se manifeste principalement avant lâge de 6 ans par des épisodes 
Table 1. Critères diagnostiques des MAI validés et disponibles à ce jour

Abréviations : IVRS = Infection des voies respiratoires supérieures; $\mathrm{CRP}=$ Protéine $\mathrm{C}$-réactive; Ig = Immunoglobuline

\begin{tabular}{|c|c|c|c|c|}
\hline & FMF"1 $^{\text {I1 }}$ & PFAPA $^{36}$ & Syndrome de Schnitzler ${ }^{90}$ & Maladie de Still+95 \\
\hline Majeurs & $\begin{array}{l}\geq 3 \text { accès typiques récurrents } \\
\text { avec fièvre }>38^{\circ} \mathrm{C} \text { et d'une } \\
\text { durée de } 12 \text { à } 72 \mathrm{~h} \text { avec } \geq 1 \\
\text { signe(s) suivant(s): } \\
\text { - Péritonite généralisée } \\
\text { - Pleurésie unilatérale ou } \\
\text { péricardite } \\
\text { - Monoarthrite de la } \\
\text { hanche, genou, cheville } \\
\text { - Fièvre isolée } \\
\text { - Accès abdominal } \\
\text { incomplet* }\end{array}$ & $\begin{array}{l}\text { 1. Fièvre récurrente débutant } \\
<5 \text { ans } \\
\text { 2. Symptômes } \\
\text { constitutionnels en } \\
\text { l'absence d'IVRS avec } \geq 1 \\
\text { signe(s) suivant(s): } \\
\text { stomatite aphteuse, adénite } \\
\text { cervicale et/ou pharyngite } \\
\text { 3. Complètement } \\
\text { asymptomatique entre les } \\
\text { épisodes } \\
\text { 4. Développement et } \\
\text { croissance normaux }\end{array}$ & $\begin{array}{l}\text { 1. Pic monoclonal IgM ou } \\
\text { IgG } \\
\text { 2. Urticaire chronique }\end{array}$ & 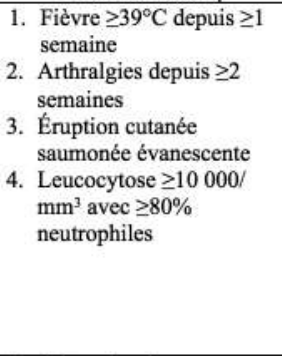 \\
\hline Mineurs & $\begin{array}{l}\text { 1. Accès incomplet* } \\
\text { touchant le thorax et/ou } \\
\text { une articulation }\end{array}$ & & $\begin{array}{l}\text { 1. Fièvre récurrente } \\
\text { 2. Signes objectifs de } \\
\text { remodelage osseux } \\
\text { anormal }+ \\
\text { 3. Infiltrat neutrophilique du } \\
\text { derme à la biopsie cutanée } \\
\text { 4. CRP }>30 \mathrm{mg} / \mathrm{L} \text { et } / \text { ou } \\
\text { neutrophiles }>10000 / \mathrm{mm}^{3}\end{array}$ & $\begin{array}{l}\text { 1. Odynophagie } \\
\text { 2. Adénopathie ou } \\
\text { splénomégalie } \\
\text { 3. Perturbation du bilan } \\
\text { hépatique } \\
\text { 4. Absence de facteur } \\
\text { rhumatoïde et anticorps } \\
\text { anti-nucléaires }\end{array}$ \\
\hline $\begin{array}{l}\text { Critères } \\
\text { d'exclusion }\end{array}$ & Aucun & Neutropénie cyclique & Aucun & $\begin{array}{l}\text { 1. Infection } \\
\text { 2. Néoplasie } \\
\text { 3. Maladies inflammatoires }\end{array}$ \\
\hline $\begin{array}{l}\text { Prérequis } \\
\text { pour } \\
\text { diagnostic } \\
\text { définitif }\end{array}$ & $\begin{array}{l}1 \text { critère majeur ou } 2 \text { critères } \\
\text { mineurs }\end{array}$ & $\begin{array}{l}4 \text { critères majeurs } \\
\text { + absence du critère } \\
\text { d'exclusion }\end{array}$ & $\begin{array}{l}2 \text { critères majeurs }+2 \text { critères } \\
\text { mineurs }(3 \text { critères mineurs si } \\
\text { pic IgG) }\end{array}$ & $\begin{array}{l}5 \text { critères incluant } \geq 2 \text { critères } \\
\text { majeurs } \\
+ \text { absence des critères } \\
\text { d'exclusion }\end{array}$ \\
\hline
\end{tabular}

Défini par au maximum 2 des signes suivants: température $<38^{\circ} \mathrm{C}$, durée plus longue ou plus courte (mais $\geq 6$ heures et $<7$ jours), péritonite

localisée ou absence de signes de péritonite en cas de crises abdominales, arthrite affectant une articulation autre que la hanche, genou ou cheville $\nmid$ †émontré par imagerie (scintigraphie osseuse ou résonance magnétique) ou augmentation de la phosphatase alcaline osseuse

„Bien que les critères de Yamaguchi soient des critères de classification (et non diagnostiques), ils peuvent aider à orienter le diagnostic.

fébriles périodiques caractérisés par une pharyngite, une aphtose buccale et/ou des adénopathies cervicales durant 3 à 6 jours (Table 1). ${ }^{35-37}$ L'inefficacité des antipyrétiques et des antibiotiques et l'excellente réponse à une prise unique de prednisone $2 \mathrm{mg} /$ $\mathrm{kg}$ confortent le diagnostic. ${ }^{38,39}$ Une majorité des cas se résout spontanément à l'adolescence, alors qu’une minorité persiste, récidive ou débute à lâge adulte. ${ }^{40-42}$

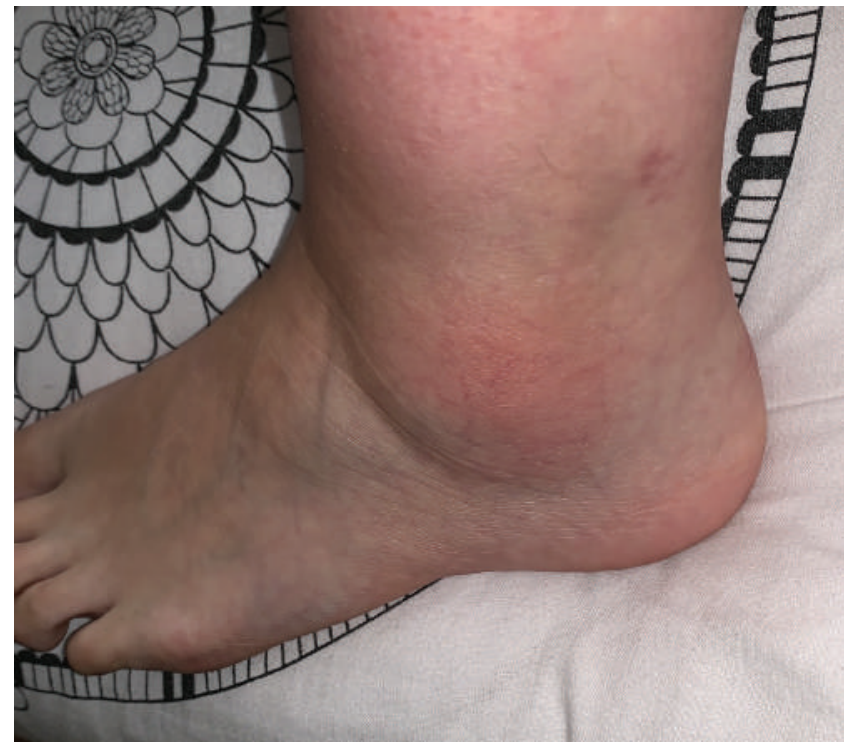

Figure 2. Pseudoérysipèle de cheville au cours d'une poussée de FMF.
B. Les MAI monogéniques ressemblant à des maladies connues

Parmi les autres MAI, certaines ressemblent à des maladies mieux connues tout en se distinguant par des signes cliniques spécifiques, un syndrome inflammatoire biologique plus important quattendu, un âge de début précoce et un caractère volontiers familial.

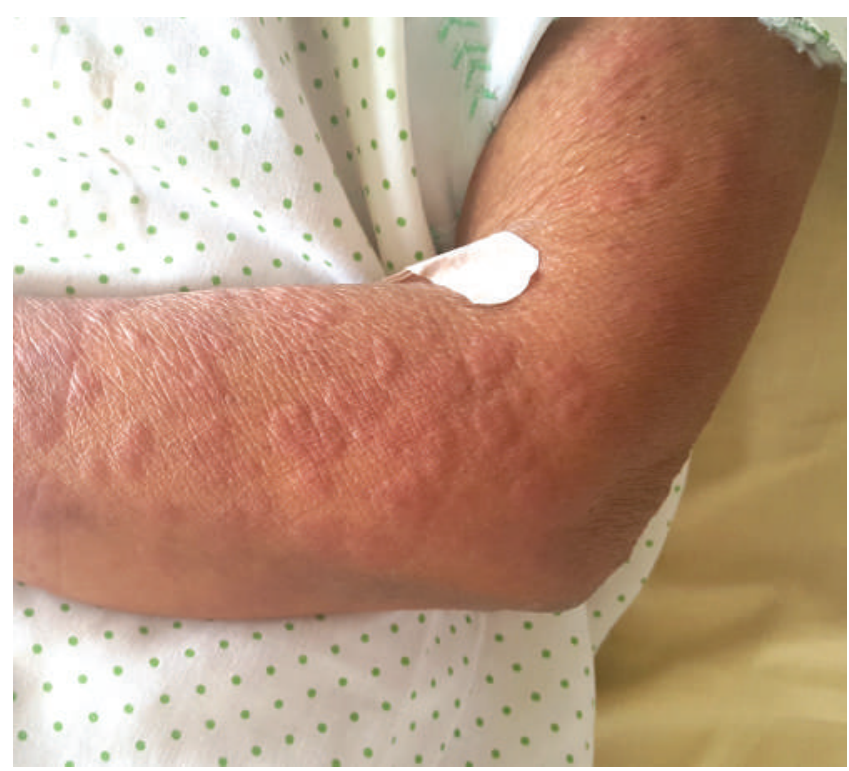

Figure 3. Lésions urticariennes du CAPS. 
Lapparition en enfance ou au début de lâge adulte d'une polyartérite noueuse avec fièvres récurrentes, livedo et manifestations neurologiques au premier plan, accompagnée dans la moitié des cas par des cytopénies et/ou un déficit immunitaire, est suggestif d'un déficit en adénosine désaminase 2 (DADA2) ${ }^{43-47}$ Le DADA2 est associé à des mutations récessives du gène $A D A 2$, qui entraîne une diminution significative de l'activité enzymatique d'ADA2. ${ }^{44}$ Celle-ci sévalue par un dosage sanguin réalisé en laboratoire spécialisé à faible coût, qui constitue un test de dépistage sensible et spécifique. ${ }^{48}$

En présence d'une aphtose bipolaire débutant dans la même tranche d'âge que celle du DADA2, on évoque une haploinsuffisance de la protéine A20 (HA20) plutôt qu'une maladie de Behçet devant des fièvres récurrentes, une atteinte digestive, des manifestations oculaires sévères à type d'uvéite antérieure ou vasculite rétinienne, une polyarthrite, un déficit immunitaire et/ou une auto-immunité clinique ou sous-clinique. ${ }^{49-53} \mathrm{Du}$ fait du caractère dominant des mutations du gène TNFAIP3 codant la protéine A20, les apparentés du premier degré sont fréquemment atteints. ${ }^{49,53}$

Une dermatose neutrophilique récidivante, notamment une hidradénite suppurée, un pyoderma gangrenosum et une acné nodulokystique sévère, constitue un signe cardinal des PAID (pour PSTPIP1-associated autoinflammatory diseases) à transmission dominante. Son sous-type le plus fréquent se complique d'une arthrite purulente aseptique. ${ }^{54-56}$ Des mutations dominantes du gène NCSTN codant la nicastrine peuvent engendrer un phénotype cutané similaire. ${ }^{57}$ Le syndrome PAAND (pour Pyrin-Associated Autoinflammation with Neutrophilic Dermatosis), associé à des mutations dominantes dans l'exon 2 du gène $M E F V$, se manifeste par des épisodes fébriles prolongés avec dermatose neutrophilique et arthromyalgies. ${ }^{58,59}$

Finalement, devant la présence de granulomes non-caséeux à la biopsie, un syndrome de Blau plutôt qu'une sarcoïdose est évoqué devant l'apparition précoce d'une éruption eczémateuse diffuse, une polyarthrite kystique déformante et une uvéite granulomateuse, et ce typiquement en l'absence d'atteinte pulmonaire. ${ }^{60}$ Ce syndrome est associé à des mutations dominantes du gène NOD $2 .^{61}$

\section{Le deuxième scénario: symptômes ayant débuté à l'âge adulte}

Le deuxième scénario représente un défi diagnostique plus grand, car la présentation clinique est souvent plus hétérogène et nécessite l'exclusion d'un large diagnostic différentiel.

\section{A. MAI monogénique à début tardif}

La FMF et le TRAPS représentent les MAI monogéniques à début tardif les plus fréquentes. ${ }^{62,63} \mathrm{Chez}$ un adulte d'origine méditerranéenne présentant une symptomatologie clinique évocatrice de FMF, ce diagnostic doit être considéré. ${ }^{64}$ Bien que l'incidence soit variable d'une population à l'autre, 10 à $30 \%$ des patients développent la FMF après lâge de 20 ans. ${ }^{65-68}$ En général, ils présentent une forme légère à modérée de la maladie, associée à des mutations $M E F V$ hétérozygotes ou moins pathogéniques que les formes à début pédiatrique. ${ }^{68-72}$ De façon similaire, le TRAPS se développe après l'âge de 20 ans dans $22 \%$ des cas, et après lâge de 30 ans dans $9 \%$ des cas. ${ }^{26}$

\section{B. MAI liée à un mosaïcisme somatique}

Les mutations somatiques sont des mutations qui surviennent pendant ou après le stade post-zygotique de l'embryogénèse. ${ }^{73,74}$ Elles conduisent au développement de deux lignées cellulaires génétiquement différentes. Plus la mutation se produit tardivement au cours de la vie, plus elle est restreinte à un nombre limité de cellules ${ }^{75}$ se traduisant par une forme de la maladie plus légère ou à début tardif. C'est le cas de certaines MAI, majoritairement les CAPS et plus rarement les syndromes TRAPS et Blau. ${ }^{19,76-82}$

Une maladie hématologique peut également engendrer une MAI par la voie du mosaïcisme. Les inflammasomes, dont les gènes sont mutés dans plusieurs MAI, sont exprimés principalement dans les cellules myéloïdes. ${ }^{83}$ Par conséquent, lorsqu'un de ces gènes auto-inflammatoires est porteur d'une mutation somatique de faible grade, une prolifération myéloïde peut entraîner l'expansion clonale et l'expression clinique de cette mutation..$^{81,84,85}$ Cette prolifération peut être due à un syndrome myélodysplasique ou myéloprolifératif, une leucémie myéloïde, ou, de façon plus occulte, à une hématopoïèse clonale de potentiel indéterminé (appelé CHIP pour Clonal hematopoiesis of indeterminate potential). Rapportée chez $15 \%$ des individus de plus de 65 ans, la CHIP réfère au stade précurseur du syndrome myélodysplasique, se manifestant par des mutations somatiques clonales sans répercussion à la numération sanguine ou médullaire. ${ }^{86,87}$

\section{MAI polygéniques}

Débutant à un âge moyen de 51 ans, ${ }^{88,89}$ le syndrome de Schnitzler associe un pic monoclonal de type IgM, ou plus rarement IgG, à une dermatose neutrophilique urticarienne (Table 1). ${ }^{90}$ Il importe toutefois d'exclure certaines maladies à présentation similaire, notamment une maladie de Still, un lupus érythémateux disséminé, une vasculite urticarienne ou encore une cryopyrinopathie par mutation somatique du gène NLRP $3{ }^{88,90}$ Plus récemment, une nouvelle MAI a été décrite en association à une gammapathie monoclonale de type IgM ou IgG, sans atteinte cutanée ou osseuse. ${ }^{91}$ Le terme MGARF (pour Monoclonal Gammopathy, Arthralgia, and Recurrent Fever) 
a été proposé, puisque ses symptômes cardinaux constituent les arthromyalgies et une fièvre récurrente.

$\mathrm{Du}$ fait de son risque accru de complications potentiellement fatales incluant le syndrome d'activation macrophagique, la maladie de Still doit être rapidement reconnue et traitée. Bien qu'elle se présente le plus souvent avant lâge de 50 ans, ${ }^{92}$ plusieurs cas à début tardif ont été rapportés. ${ }^{93,94}$ En l'absence d'un signe pathognomonique, des critères de classification peuvent aider au diagnostic; les mieux connus sont ceux de Yamaguchi (Table 1 ), avec une valeur prédictive positive et négative de $94,5 \%$ et 99,3\% respectivement. ${ }^{95,96}$

Tel que mentionné dans le premier scénario, le syndrome de PFAPA peut parfois récidiver ou débuter à l'âge adulte. Le patient typique est âgé de moins de 50 ans et présente des symptômes similaires au PFAPA de l'enfant. ${ }^{41,97,98}$ Les crises semblent toutefois plus longues, moins fréquentes et plus souvent accompagnées d’arthromyalgies, manifestations muco-cutanées et céphalées. ${ }^{97,98}$ À l'inverse des enfants, une majorité répondent aux anti-inflammatoires non-stéroïdiens lors des crises et à la colchicine en traitement préventif, et très peu répondent à un traitement chirurgical par amygdalectomie. ${ }^{42,97-99}$

\section{MAI inclassées}

Finalement, certains patients présentent un tableau de fièvre récurrente évocateur d'un processus auto-inflammatoire sans toutefois correspondre à l'une des MAI héréditaires ou acquises décrites ci-haut. Cela correspond à 10 à $15 \%$ des MAI. ${ }^{100-102}$ Certains évolueront au fil du temps en une MAI bien définie, alors que d'autres non.

\section{La démarche diagnostique}

À ce jour, très peu de MAI possèdent des critères diagnostiques bien établis (Table 1). Par conséquent, leur diagnostic repose sur l'identification déléments cliniques et paracliniques concordants, l'exclusion de leur diagnostic différentiel et, en cas de maladie monogénique, une analyse génétique compatible.

\section{L'histoire et l'examen physique}

En cas de suspicion de MAI, la réalisation d'un interrogatoire et d'un examen physique méticuleux sont essentiels, du fait que cette maladie évolue par poussées et que le patient est donc souvent asymptomatique au moment de la visite médicale.

Larbre généalogique constitue le point de départ de l'évaluation. Remontant jusqu'aux grands-parents, il permet de déterminer sur 3 générations ses origines ethniques et géographiques, la présence de consanguinité et le mode de transmission de la maladie. La survenue de la maladie à chaque génération oriente vers une transmission dominante (verticale), alors que la présence de consanguinité ou de maladie chez la fratrie évoque une transmission récessive (horizontale) (Figure 4).

La deuxième étape consiste à déterminer si le tableau clinique est en faveur d'une MAI. Celle-ci se manifeste habituellement par des épisodes inflammatoires intermittents, bien stéréotypés, auto-résolutifs et alternant avec des périodes asymptomatiques. Outre la fièvre qui est rarement nue, les atteintes muco-cutanées, digestives, musculosquelettiques et des séreuses sont les plus fréquentes. La complétion du score AIDAI (Auto-Inflammatory Diseases Activity Index) sur 6 mois peut aider à préciser le tableau clinique. ${ }^{103,104}$ Disponible en ligne et validé dans le suivi des 4 MAI historiques, ce score comprend 12 symptômes à évaluer de façon dichotomique sur une base quotidienne. La résistance au traitement par anti-inflammatoires non-stéroïdiens, corticostéroïdes et immunosuppresseurs conventionnels et biologiques peut parfois faire évoquer une MAI, qui répond généralement mieux aux agents ciblant les interleukines (IL) 1 et 6 et parfois le TNF. ${ }^{105}$

La troisième étape vise à identifier le type de MAI (Figure 5). Il faut déterminer avec précision lâge de début des symptômes.

\begin{tabular}{|c|c|c|c|}
\hline & $<18$ ans & $\geq 18$ ans & $\begin{array}{l}\text { Exemples d'arbre } \\
\text { généalogique }\end{array}$ \\
\hline AD & $\begin{array}{l}\text { TRAPS** } \\
\text { CAPS** } \\
\text { HA20 } \\
\text { PAID } \\
\text { PAAND }\end{array}$ & $\begin{array}{l}\text { TRAPS** } \\
\text { CAPS** }\end{array}$ & 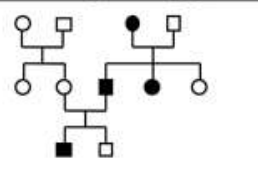 \\
\hline $\mathbf{A R}$ & $\begin{array}{l}\text { FMF*** } \\
\text { MVK* } \\
\text { DADA2* }\end{array}$ & FMF*** & 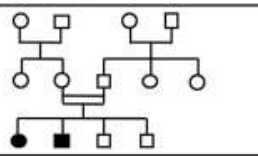 \\
\hline
\end{tabular}

Figure 4. Schématisation des principales MAI monogéniques selon leur mode de transmission et leur

${ }^{* * *}=$ très amyloïdogène; ${ }^{*}$ * $=$ modérément amyloïdogène; ${ }^{*}=$ peu amyloïdogène

Abréviations: $A D=$ Autosomique dominant; $A R=$ Autosomique récessif

Cette figure présente les MAI monogéniques les plus fréquentes et ne se veut pas exhaustive. 
Ainsi, un début précoce est en faveur d'une MAI monogénique alors qu'un début plus tardif est évocateur de certaines MAI monogéniques (TRAPS et FMF), de maladies polygéniques et de MAI liées à un mosaïcisme somatique. Il est également important détablir la durée, la fréquence et les facteurs déclenchant des épisodes. Une évolution sur plusieurs semaines ou parfois chronique s'observe dans la maladie de Still, le TRAPS et les CAPS sévères; une durée inférieure à 7 jours oriente vers la FMF, le MKD et le PFAPA. ${ }^{106}$ Finalement, alors que le stress physique ou physiologique, les infections et les menstruations peuvent exacerber les MAI, la vaccination et le froid provoquent plus spécifiquement des poussées de MKD et de CAPS de façon respective. ${ }^{23,31}$

\section{Les examens paracliniques}

Les examens paracliniques servent à confirmer le processus inflammatoire systémique, obtenir des signes objectifs en faveur de la MAI suspectée et exclure les diagnostics alternatifs.

En premier lieu, il faut déterminer si la protéine C-réactive (CRP) sélève en cas de crise. Dans les MAI, les marqueurs inflammatoires sélèvent lors des poussées et se normalisent ou du moins diminuent entre celles-ci. Un diagnostic de MAI peut seulement être évoqué en présence d’au minimum 3 épisodes inflammatoires avec CRP élevée sur une période de 6 mois ou plus. ${ }^{107} \mathrm{Si}$ son dosage est disponible, la protéine amyloïde A sérique (SAA) devrait être mesurée à au moins une reprise. La SAA est une apolipoprotéine impliquée dans la réaction de

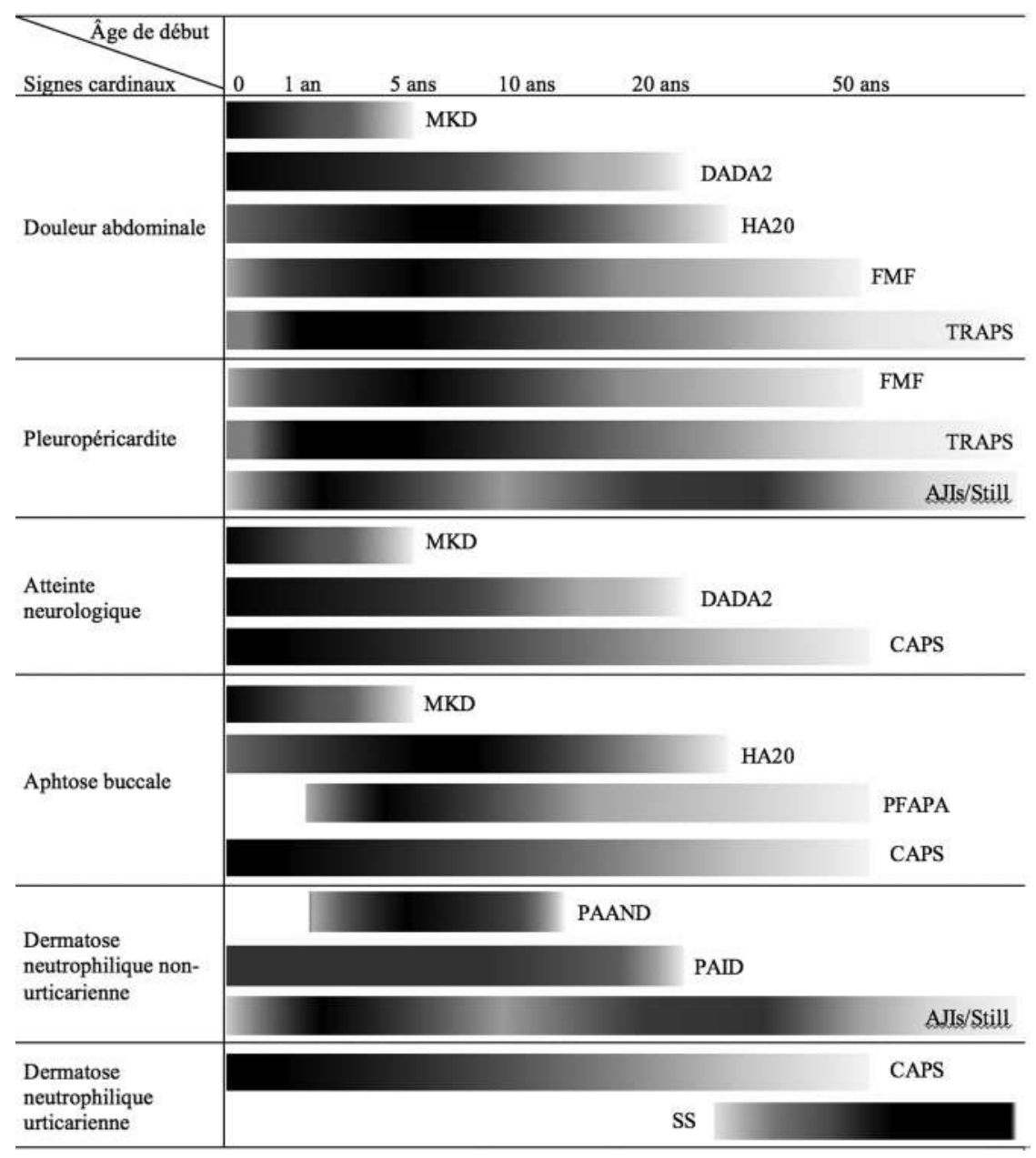

Figure 5. Présentation des principales MAI monogéniques selon leurs signes cliniques les plus fréquents et leur âge de début

La couleur noire représente une incidence élevée, la couleur grise une incidence modérée, et la couleur blanche une incidence faible de la maladie selon l'âge de début.

Abréviations: Alls = Arthrite juvénile idiopathique systémique; SS = Syndrome de Schnitlzer

Cette figure présente les MAI monogéniques les plus fréquentes et ne se veut pas exhaustive 
phase aigüe, augmentant conjointement à la CRP dans toute maladie inflammatoire. ${ }^{107}$ Bien qu'elle constitue les dépôts fibrillaires de l'amylose AA, un taux élevé de SAA demeure tout à fait non-spécifique; son intérêt principal est d'identifier la minorité de patients chez qui la corrélation entre la CRP et la SAA est imparfaite, la SAA étant un marqueur inflammatoire plus sensible que la CRP. ${ }^{108-110}$

Chez l'adulte comme chez l'enfant, un processus autoimmun, néoplasique ou infectieux et un déficit immunitaire peuvent simuler une MAI. Il est donc nécessaire de rechercher les signes en faveur de ces maladies. De façon générale, les maladies dorigine non-auto-inflammatoire se manifestent par une symptomatologie chronique plutôt que par poussées se résolvant spontanément. Une maladie auto-immune est évoquée devant la présence de marqueurs d'auto-immunité et un syndrome inflammatoire biologique chronique et moins marqué que celui retrouvé chez les MAI, avec notamment peu ou pas de leucocytose à polynucléaires neutrophiles. Elle s'apparente toutefois à un type particulier de MAI appelé interféronopathie, qui se distingue par un âge de début très précoce, une histoire familiale souvent positive et plusieurs atypies cliniques. À la différence des processus néoplasiques, les patients avec MAI sont généralement bien portants en dehors des crises. Une instabilité hémodynamique est rarement observée et, si présente, oriente plutôt vers un processus infectieux, tout comme une réponse positive aux agents antimicrobiens. Néanmoins, les syndromes lymphoprolifératifs, notamment la maladie de Castleman multicentrique, et certaines infections peuvent se présenter avec des fièvres récurrentes.

Par conséquent, en cas de suspicion de MAI, les investigations de base sont les suivantes : une formule sanguine complète afin de documenter une leucocytose à polynucléaires neutrophiles; un dosage de la CRP en crise afin de confirmer son caractère inflammatoire, et hors crise afin d'exclure la persistance d'une inflammation interictale; un dosage de la SAA si possible afin de confirmer sa corrélation avec la CRP; un frottis sanguin à la recherche d'une hémopathie; une électrophorèse des protéines sérique pour rechercher une gammapathie monoclonale et une hypogammaglobulinémie, ainsi qu'une évaluation de la fonction rénale et de la protéinurie afin d'exclure une amylose AA concomitante. Selon l'évaluation clinique, des examens supplémentaires tels qu'une imagerie thoraco-abdomino-pelvienne, des endoscopies digestives ou un bilan microbiologique, autoimmun ou d'immunodéficience peuvent saavérer nécessaires. La biopsie d'une éruption cutanée peut permettre de restreindre le diagnostic différentiel, notamment en cas de dermatose neutrophilique.

Selon le type de MAI pressenti, des examens spécifiques, telle la mesure de l'activité sérique d'ADA2 pour le DADA2 et de l'acidurie mévalonique en crise pour le $\mathrm{MKD}$, servent à affermir la suspicion clinique et guider l'analyse génétique. Bien que cette analyse génétique puisse permettre de confirmer le diagnostic d'une MAI monogénique ou liée au mosaïcisme somatique, elle ne peut l'exclure. ${ }^{111,112}$ À ce jour, elle s'avère négative chez la majorité des adultes avec MAI suspectée. ${ }^{113-115}$ Le diagnostic s'appuie donc avant tout sur la clinique. Par conséquent, l'amorce d'un traitement ne doit pas être retardée en attendant les résultats de cette analyse. Néanmoins, deux techniques de séquençage sont maintenant disponibles : Sanger et nouvelle génération (NGS pour Next Generation Sequencing). La première permet l'analyse d'un ou de quelques gènes à la fois avec une haute spécificité mais une sensibilité limitée; elle est plus rapide et moins coûteuse. ${ }^{116}$ La deuxième permet l'analyse concomitante de plusieurs gènes avec une meilleure sensibilité mais une spécificité limitée par l'identification de nombreux variants génétiques d'interprétation parfois difficile. ${ }^{116,117}$ Par conséquent, devant l'apport majoritairement limité et la complexité de l'analyse génétique, il est préférable de contacter ou référer le patient à un expert en MAI.

\section{L'approche thérapeutique}

Compte tenu de la rareté des MAI et de leurs particularités thérapeutiques, leur prise en charge doit se faire conjointement à des experts en la matière. L'objectif du traitement est double: maintenir une qualité de vie acceptable et réduire les risques de complications, notamment l'amylose AA. ${ }^{118}$ En cas de poussées inflammatoires peu fréquentes et brèves, de courtes cures de corticostéroïdes peuvent s’avérer utiles. ${ }^{119}$ Efficace, bien tolérée et peu coûteuse, la colchicine est administrée comme traitement de fond de première intention chez plusieurs MAI, dont la FMF. ${ }^{120-}$

${ }^{122}$ Compte tenu de son délai d'action, l'efficacité de la colchicine sévalue après un minimum de 3 à 6 mois d’observance adéquate au traitement. ${ }^{123}$ Le recours aux biothérapies contre l'IL-1, l'IL-6 et le TNF est parfois nécessaire selon le type de MAI. ${ }^{118,124}$

Les MAI ont un impact important sur la qualité de vie des patients, notamment d'un point de vue social et professionnel en lien avec une fatigue chronique, des troubles de concentration, un absentéisme fréquent et la mauvaise compréhension de leur maladie par l'entourage autant personnel que médical. ${ }^{32,125-128} \mathrm{Il}$ est donc primordial de tenir compte de ces aspects psychosociaux lors de la prise en charge du patient.

\section{Le suivi}

Lamylose AA demeure un mode de présentation des MAI, en lien avec un important retard diagnostique entraînant une inflammation chronique ou récurrente. ${ }^{129}$ Le risque d’amylose est variable et parfois difficile à individualiser d'un patient à l'autre, puisqu'il dépend du potentiel amyloïdogène de chaque MAI, 
de la présence de maladies inflammatoires concomitantes ainsi que de facteurs environnementaux et génétiques. ${ }^{130} \mathrm{Il}$ demeure néanmoins élevé chez les MAI non-traitées, avec une incidence supérieure à $10 \%$ au cours des 4 fièvres héréditaires historiques. ${ }^{130}$ Latteinte rénale constitue sa manifestation principale et est retrouvée chez plus de $95 \%$ des patients. ${ }^{129,131}$ Son pronostic est réservé, avec une survie rénale d'environ 5 ans en l'absence de traitement anti-inflammatoire adéquat. ${ }^{132,133}$ Il convient donc d'effectuer une surveillance au moins annuelle des marqueurs inflammatoires, de la fonction rénale et de la protéinurie chez les patients dont la MAI est bien contrôlée, et de façon plus rapprochée chez les patients moins bien contrôlés.

\section{Conclusion}

L'univers des MAI est en pleine expansion, avec la découverte à chaque année de nouvelles maladies impliquant l'hyper-activation du système immunitaire inné. Chez l'adulte, des fièvres récurrentes accompagnées de sérites et/ou de signes muco-cutanés, digestifs et musculosquelettiques doivent faire évoquer une MAI. Le partage des connaissances concernant ces maladies permettra progressivement de réduire leur délai diagnostique et optimiser leur prise en charge, avec l'objectif ultime d'aider les patients à reprendre contrôle sur leur vie et prévenir le développement de complications morbides et potentiellement mortelles.

\section{Points clé à retenir}

1. Les MAI résultent d'un défaut héréditaire ou acquis de l'immunité innée.

2. Un minimum de 3 épisodes avec CRP élevée, dont la majorité sont accompagnés de fièvre et de symptômes stéréotypés, sont requis pour faire évoquer une MAI.

3. L’arbre généalogique, les antécédents familiaux, l'histoire de la maladie, l'examen physique et la réalisation d'un bilan sanguin et urinaire de base sont les éléments essentiels à l'évaluation d'une MAI suspectée.

4. La décision de réaliser une analyse génétique doit idéalement être prise de concert avec un expert en la matière.

5. Lamylose inflammatoire (AA) est la complication la plus grave des MAI, et doit être dépistée annuellement par la créatininémie et la protéinurie.

Nous déclarons que le manuscrit n’a jamais été soumis à des fins de publication.

Conflits d'intérêts : aucun concernant cet article. SGL et GG ont perçu par le passé des frais d’orateurs ou de déplacements des laboratoires suivants : SOBI, NOVARTIS et BMS.

\section{Acronymes (par ordre alphabétique)}

FMF: $\quad$ Fièvre méditerranéenne familiale

CAPS: Cryopyrin-associated periodic syndrome

CHIP: Clonal hematopoiesis of indeterminate potential

DADA2: Déficit en adénosine désaminase 2

HA20: Haplo-insuffisance de la protéine A20

MGARF: Monoclonal Gammopathy, Arthralgia, and Recurrent Fever

MKD: $\quad$ Mevalonate kinase deficiency

PAAND: Pyrin-Associated Autoinflammation with Neutrophilic Dermatosis

PAID: $\quad$ PSTPIP1-associated autoinflammatory diseases

PFAPA: Periodic fever, aphthous stomatitis, pharyngitis and adenitis

TRAPS: TNF receptor-associated periodic fever syndrome

\section{Bibliographie}

1. Ben-Chetrit E, Gattorno M, Gui A, et al. Consensus proposal for taxonomy and definition of the autoinflammatory diseases (AIDs): a Delphi study. Ann Rheum Dis. 2018; 77(11): 1558-1565.

2. Ben-Chetrir E, Touitou I. Familial Mediterranean Fever in the World. Arthritis Rheum. 2009; 61(10): 1447-1453.

3. Zhang S. Natural history of mevalonate kinase deficiency: a literature review. Pediatr Rheumatol Online J. 2016; 14(1): 30.

4. Hausmann JS, Lomax KG, Shapiro A, et al. The patient journey to diagnosis and treatment of autoinflammatory diseases. Orphanet J Rare Dis. 2018; 13(1): 156.

5. Chuamanochan M, Weller K, Feist E, et al. State of care for patients with systemic autoinflammatory diseases - Results of a tertiary care survey. World Allergy Organ J. 2019; 12(3): 100019.

6. David K, Yougstein T, Lane T, et al. Retrospective Analysis of Adult Referrals to the Periodic Fever Service at the National Amyloidosis Centre: Increasing Recognition of Adult-Onset Genetic Autoinflammatory Disease. Rheumatology. 2016; 55(1): i47.

7. McGonagle D, McDermott MF. A proposed classification of the immunological diseases. PLoS Med 3.8 (2006): e297.

8. The French FMF Consortium. A candidate gene for familial Mediterranean fever. Nat Genet. 1997; 17: 25-31.

9. The international FMF Consortium. Ancient Missense Mutations in a New Member of the RoRet Gene Family Are Likely to Cause Familial Mediterranean Fever. Cell Press. 1997; 90: 797-807.

10. Ben-Chetrit E, Levy M. Familial Mediterranean fever. Lancet. 1998; 351(9103): 659-664.

11. Livneh A, Langevitz $P$, Zemer D, et al. Criteria for the diagnosis of familial mediterranean fever. Arthritis Rheum. 1997; 40(10): 1879-1885.

12. Barzilai A, Langevitz P, Goldberg I, et al. Erysipelas-like erythema of familial Mediterranean fever: clinicopathologic correlation. J Am Acad Dermatol. 2000; 42 (5 Pt 1): 791-5.

13. Majeed HA, Al-Qudah AK, Qubain H, et al. The clinical patterns of myalgia in children with familial Mediterranean fever. Semin Arthritis Rheum. 2000; 30(2): 138.

14. Livneh A, Langevitz P, Zemer D, et al. The changing face of Familial Mediterranean Fever. Semin Arthritis Rheum. 1996; 26(3): 612-627.

15. Altunoğlu A, Erten S, Canoz MB, et al. Phenotype 2 Familial Mediterranean Fever: Evaluation of 22 Case Series and Review of the Literature on Phenotype 2 FMF. Ren Fail. 2013; 35(2): 226-30. 
16. Georgin-Lavialle S, Stankovic K, Cez A, et al. Amylose AA secondaire à la fièvre méditerranéenne familiale : à propos de 38 cas. Rev Med Interne. 2016; 37(2): A119-A120.

17. Hoffman HM, Muelle JL, Broide DH, et al. Mutation of a new gene encoding a putative pyrin-like protein causes familial cold autoinflammatory syndrome and Muckle-Wells syndrome. Nat Genet. 2001; 29(3): 301-5.

18. Aksentijevich I, Nowak M, Mallah M, et al. De novo CIAS1 mutations, cytokine activation, and evidence for genetic heterogeneity in patients with neonatal-onset multisystem inflammatory disease (NOMID): a new member of the expanding family of pyrin-associated autoinflammatory diseases. Arthritis Rheum. 2002; 46(12): 3340-8.

19. Labrousse M, Kevorkian-Verguet C, Boursier G, et al. Mosaicism in autoinflammatory diseases: Cryopyrin-associated periodic syndromes (CAPS) and beyond. A systematic review. Crit Rev Clin Lab Sci. 2018; 55(6): 432-442.

20. Kuemmerle-Deschner J. CAPS - pathogenesis, presentation and treatment of an autoinflammatory disease. 2017; 37(4).

21. Quartier P, Rodrigues F, Gerogin-Lavialle S. Cryopyrinopathies. La Revue de Médecine Interne. 2018; 39(4): 287-296.

22. Koné-Paut I. Syndrome périodique lié à la cryopyrine : CAPS vu à lâge adulte. Rev Med Interne. 2015; 36(4): 277-282.

23. Levy R, Gérard L, Kuemmerle-Deschner J, et al. Phenotypic and genotypic characteristics of cryopyrin-associated periodic syndrome: a series of 136 patients from the Eurofever Registry. Ann Rheum Dis. 2015; 74: 2043-2049.

24. Parker T, Keddie S, Kidd D, et al. Neurology of the cryopyrin-associated periodic fever syndrome. Eur J Neurol. 2016; 73: 1145-1151.

25. McDermott MF, Aksentijevich I, Galon J, et al. Germline mutations in the extracellular domains of the $55 \mathrm{kDa}$ TNF receptor, TNFR1, define a family of dominantly inherited autoinflammatory syndromes. Cell. 1999; 97(1): 133-144.

26. Lachmann HJ, Papa R, Gerhold K, et al. The phenotype of TNF receptorassociated autoinflammatory syndrome (TRAPS) at presentation: a series of 158 cases from the Eurofever/EUROTRAPS international registry. Ann Rheum Dis. 2013; 73(12): 2160-2167.

27. Lachmann HJ. Periodic fever syndromes. Best Pract Res Clin Rheumatol. 2017; 31(4): 596-609.

28. Houten SM, van Woerden CS, Wijburg FA, et al. Carrier frequency of the V377I (1129G $>$ A) MVK mutation, associated with Hyper-IgD and periodic fever syndrome, in the Netherlands. Eur J Hum Genet. 2003; 11(2): 196-200.

29. Jeyaratnam J, Ter Haar NM, de Sain-van der Velden MG, et al. Diagnostic Value of Urinary Mevalonic Acid Excretion in Patients with a Clinical Suspicion of Mevalonate Kinase Deficiency (MKD). JIMD Rep. 2016; 27: $33-38$.

30. Ammouri W, Cuisset L, Rouaghe S, et al. Diagnostic value of serum immunoglobulinaemia $\mathrm{D}$ level in patients with a clinical suspicion of hyper IgD syndrome. Rheumatol. 2007; 46(10): 1597-1600.

31. Ter Haar NM, Jeyaratnam J, Lachmann HJ, Jeyaratnam J, et al. The Phenotype and Genotype of Mevalonate Kinase Deficiency: A Series of 114 Cases From the Eurofever Registry. Arthritis Rheumatol. 2016; 68(11): 2795-2805.

32. Van der Hilst JC, Bodar EJ, Barron KS, et al. Long-Term Follow-Up, Clinical Features, and Quality of Life in a Series of 103 Patients With Hyperimmunoglobulinemia D Syndrome. Medicine (Baltimore). 2008; 87(6): 301-310.

33. Durel CA, Aouba A, Bienvenu B, et al. Observational Study of a French and Belgian Multicenter Cohort of 23 Patients Diagnosed in Adulthood With Mevalonate Kinase Deficiency. Medicine (Baltimore). 2016; 95(11): e3027.

34. Wekell P, Karisson A, Berg S, et al. Review of autoinflammatory diseases, with a special focus on periodic fever, aphthous stomatitis, pharyngitis and cervical adenitis syndrome. 2016; 105: 1140-1151.

35. Marshall GS, Edwards KM, Butler J, et al. Syndrome of periodic fever, pharyngitis, and aphthous stomatitis. J Pediatr. 1987; 110(1): 43-46.
36. Thomas KT, Feder HM Jr, Lawton AR, et al. Periodic fever syndrome in children. J Pediatr. 1999; 135: 15-21.

37. Vanoni F, Caorsi R, Aeby S, et al. Towards a new set of classification criteria for PFAPA syndrome. Pediatr Rheumatol Online J. 2018; 16(1): 60.

38. Harel L, Hashkes PJ, Lapidus S, et al.The First International Conference on Periodic Fever, Aphthous Stomatitis, Pharyngitis, Adenitis Syndrome. J Pediatr. 2018; 193: 265-274.e3.

39. Hofer M, Pillet P, Cochard MM, et al. International periodic fever, aphthous stomatitis, pharyngitis, cervical adenitis syndrome cohort: description of distinct phenotypes in 301 patients. Rheumatology. 2014; 53(6): 1125-1129.

40. Wurster VM, Carlucci JG, Feder HM, et al. Long-term follow-up of children with periodic fever, aphthous stomatitis, pharyngitis, and cervical adenitis syndrome. J Pediatr. 2011; 159(6): 958-964.

41. Padeh S, Stoffman N, Berkun Y. Periodic fever accompanied by aphthous stomatitis, pharyngitis and cervical adenitis syndrome (PFAPA syndrome) in adults. Isr Med Assoc J. 2008; 10: 358-360.

42. Cantarini L, Vitale A, Bartolomei B, et al. Diagnosis of PFAPA syndrome applied to a cohort of 17 adults with unexplained recurrent fever. Clin Exp Rheumatol. 2012; 30(2): 269-271.

43. Zhou Q, Yang D, Ombrello AK, et al. Early-onset stroke and vasculopathy associated with mutations in ADA2. NEJM. 2014; 370(10): 911-920.

44. Rama M, Duflos C, Melki I, et al. A decision tree for the genetic diagnosis of deficiency of adenosine deaminase 2 (DADA2): a French reference centres experience. Eur J Hum Genet. 2018; 26: 960-971.

45. Navon Elkan P, Pierce SB, Segel R, et al. Mutant adenosine deaminase 2 in a polyarteritis nodosa vasculopathy. NEJM. 2014; 370(10): 921-931.

46. Lee P. Vasculopathy, Immunodeficiency, and Bone Marrow Failure: The Intriguing Syndrome Caused by Deficiency of Adenosine Deaminase 2. Front Pediatr. 2018; 6: 282.

47. Meyts I, Aksentijevich I. Deficiency of Adenosine Deaminase 2 (DADA2) Updates on the Phenotype, Genetics, Pathogenesis, and Treatment. J Clin Immunol. 2018; 38(5): 569-578.

48. Ozen S, Bilginer Y, Batu ED, et al. A monogenic disease with a variety of phenotypes: deficiency of adenosine deaminase. J Rheumatol. 2019;: doi: 10.3899/jrheum.181384. [Epub ahead of print].

49. Zhou Q, Wang H, Schwartz DM, et al. Loss-of-function mutations in TNFAIP3 leading to A20 haploinsufficiency cause an early-onset autoinflammatory disease. Nat Genet. 2016; 48: 67-73.

50. Aeschlimann FA, Batu ED, Canna SW, et al. A20 haploinsufficiency (HA20): clinical phenotypes and disease course of patients with a newly recognised NF-kB-mediated autoinflammatory disease. Ann Rheum Dis. 2018; 77(5): 728-735.

51. Kadowaki T, Ohnishi H, Kawamoto N, et al. Haploinsufficiency of A20 causes autoinflammatory and autoimmune disorders. J Allergy Clin Immunol. 2018; 141(4): 1485-1488.e11.

52. Berteau F, Rouviere B, Delluc A, et al Autosomic dominant familial Behçet disease and haploinsufficiency A20: A review of the literature. Autoimmun Rev. 2018; 17(8): 809-815.

53. Tsuchida N, Kirino Y, Soejima Y, et al. Haploinsufficiency of A20 caused by a novel nonsense variant or entire deletion of TNFAIP3 is clinically distinct from Behçet's disease. Arthritis Res Ther. 2019; 21(1): 137.

54. Holzinger D, Fassl SK, de Jager W, et al. SdJWea. Single amino acid charge switch defines clinically distinct proline-serine-threonine phosphataseinteracting protein 1 (PSTPIP1)-associated inflammatory diseases. J Allergy Clin Immunol. 2015; 136(5): 1337-1345.

55. Holzinger D, Roth J. Alarming consequences - autoinflammatory disease spectrum due to mutations in proline-serine-threonine phosphataseinteracting protein 1. Curr Opin Rheumatol. 2016; 28(5): 550-559.

56. Lindor NM, Arsenault TM, Solomon H, et al. A new autosomal dominant disorder of pyogenic sterile arthritis, pyoderma gangrenosum, and acne: PAPA syndrome. Mayo Clin Proc. 1997; 72(7): 611-615.

57. Sonbol H, Duchatelet S, Miskinyte S, et al. PASH syndrome (pyoderma gangrenosum, acne and hidradenitis suppurativa): a disease with genetic heterogeneity. Br J Dermatol. 2018; 178(1): e17-e18. 
58. Masters SL, Lagou V, Jeru I, et al. Familial autoinflammation with neutrophilic dermatosis reveals a regulatory mechanism of pyrin activation. Sci Transl Med. 2016; 8(332): 332ra45.

59. Moghaddas F, Llamas R, De Nardo D, et al. A novel Pyrin-Associated Autoinflammation with Neutrophilic Dermatosis mutation further defines 14-3-3 binding of Pyrin and distinction to Familial Mediterranean Fever. Ann Rheum Dis. 2017; 76(12): 2085-2094.

60. Caso F, Galozzi P, Costa L, et al. Autoinflammatory granulomatous diseases: from Blau syndrome and early-onset sarcoidosis to NOD2-mediated disease and Crohn's disease. RMD Open. 2015; 1(1): e000097.

61. Miceli-Richard C, Lesage S, Ryboiad M, et al. CARD15 mutations in Blau syndrome. Nat Genet. 2001; 29(1): 19-20.

62. Muscari I, Lacoponi F, Cantarini L, et al. The diagnostic evaluation of patients with potential adult-onset autoinflammatory disorders: Our experience and review of the literature. Autoimmun Rev. 2012; 12(1): 10-13.

63. Cantarini L, Vitale A, Lucherini OM, et al. Childhood versus adulthoodonset autoinflammatory disorders: myths and truths intertwined. Rheumatismo. 2013; 65(2): 55-62.

64. Federici L, Rittore-Domingo C, Koné-Paut I, et al. A decision tree for genetic diagnosis of hereditary periodic fever in unselected patients. Ann Rheum Dis. 2006; 65(11): 1427-1432.

65. Sohar E, Gafni J, Pras M, et al. Familial Mediterranean fever. A survey of 470 cases and review of the literature. Am J Med. 1967; 43(2): 227-253.

66. Üreten K, Gönülalan G, Akbal E, et al. Rheumatology International. 2010; 30(7): 911-915.

67. Kriegshauser G, Enko D, Hayrapetyan H, et al. Genotypic diversity observed within a large cohort of Armenian patients with late-onset familial Mediterranean fever. Genet Med. 2018; 20: 1583-1588.

68. Yasar Bilge NS, Sari I, Solmaz D, et al. Comparison of early versus late onset familial Mediterranean fever. Int J Rheum Dis, 21: 880-884. Int J Rheum Dis. 2018; 21: 880-884.

69. Tamir N, Langevitz P, Zemer D, et al. Late onset familial Mediterranean fever (FMF): a subset with distinct clinical, demographic, and molecular genetic characteristics. Am J Med Genet. 1999; 87: 30-35.

70. Sayarlioglu M, Cefle A, Inanc M, et al. Characteristics of patients with adultonset familial Mediterranean fever in Turkey: analysis of 401 cases. Int J Clin Pract. 2005; 59(2): 202-205.

71. Nobakht H, Zamani F, Ajdarkosh H, et al. Adult-Onset Familial Mediterranean Fever in Northwestern Iran; Clinical Feature and Treatment Outcome. Middle East J Dig Dis. 2011; 3(1): 50-55.

72. Marek-Yagel D, Berkun Y, Padeh S, et al. Clinical disease among patients heterozygous for familial mediterranean fever. Arthritis Rheumatol. 2009; 60(6): 1862-1866.

73. Hoffman HM, Broderick L. Editorial: It Just Takes One: Somatic Mosaicism in Autoinflammatory Disease. Arthritis Rheumatol. 2017; 69(2): 253-256.

74. Stoffels M, Kastner DL. Old Dogs, New Tricks: Monogenic Autoinflammatory Disease Unleashed. Annu Rev Genomics Hum Genet. 2016; 17(1): 245-272.

75. Forsberg LA, Gisselsson D, Dumanski JP. Mosaicism in health and disease clones picking up speed. Nat Rev Genet. 2017; 18(2): 128-142.

76. De Inocencio J, Mensa-Vilaro A, Tejada-Palacios P, et al. Somatic NOD2 mosaicism in Blau syndrome. J Allergy Clin Immunol. 2015; 136(2): 484487.e2.

77. Mensa-Vilaro A, Cham WT, Tang SP, et al. Brief report: first identification of intrafamilial recurrence of Blau syndrome due to gonosomal NOD2 mosaicism. Arthritis Rheumatol. 2016; 68(4): 1039-1044.

78. Mensa-Vilaro A, Teresa-Bosque M, Magri G, et al. Late onset cryopyrinassociated periodic syndrome due to myeloid-restricted somatic NLRP3 mosaicism. Arthritis Rheumatol. 2016; 68(12): 3035-3041.

79. Zhou Q, Aksentijevich I, Wood GM, et al. Brief report: cryopyrin-associated periodic syndrome caused by a myeloid-restricted somatic NLRP3 mutation. Arthritis Rheumatol. 2015; 67(9): 2482-2486.
80. Rowczenio D, Omoyinmi E, Trojer H, et al. First case of somatic mosaicism in TRAPS caused by a novel 24 nucleotides deletion in the TNFRSF1A gene. Pediatr Rheumatol Online J. 2015; 13(Suppl 1): O60.

81. Rowczenio DM, Gomes SM, Arostegui JI, et al. Late-Onset CryopyrinAssociated Periodic Syndromes Caused by Somatic NLRP3 Mosaicism-UK Single Center Experience. Front Immunol. 2017; 8: 1410.

82. Kontzias A, Zarabi S, Calabrese C, et al. Late onset tumour necrosis factor receptor (TNFR)-associated periodic syndrome (TRAPS) caused by somatic tnfrsf1 mosaicism. Ann Rheum Dis. 2018; 77: 1670-1671.

83. Jha S, Brickey WJ, Ting JP. Inflammasomes in Myeloid Cells: Warriors Within. Microbiol Spectr. 2017; 5(1): 10.

84. Shinar Y, Tohami T, Livneh A, et al. Acquired familial Mediterranean fever associated with a somatic MEFV mutation in a patient with JAK2 associated post-poly- cythemia myelofibrosis. Orphanet J Rare Dis. 2015; 10: 86.

85. Shinar Y, Cohen R, Marcu V, et al. Severe prognosis of late onset CAPS associated with age-related clonal hematopoiesis. Présentation de poster au 10ème congrès de l'ISSAID. Avril 2019.

86. Valent P, Kern W, Hoermann G, et al. Clonal Hematopoiesis with Oncogenic Potential (CHOP): Separation from CHIP and Roads to AML. Int J Mol Sci. 2019; 20: 789.

87. Steensma DP, Bejar R, Jaiswal S, et al. Clonal hematopoiesis of indeterminate potential and its distinction from myelodysplastic syndromes. Blood. 2015; 126(1): 9-16.

88. Koning HD. Schnitzler's syndrome: lessons from 281 cases. Clin Transl Allergy. 2014; 4(1): 41.

89. Rowczenio DM, Pathak S, Arostegui JI. Molecular genetic investigation, clinical features, and response to treatment in 21 patients with Schnitzler syndrome. Blood. 2018; 131(9): 974-981.

90. Simon A, Asli B, Braun-Falco M, et al. Schnitzler's syndrome: diagnosis, treatment, and follow-up. Allergy. 2013; 68(5): 562- 568.

91. Terré A, Talbot A, Louvrier C, et al. Monoclonal gammopathy, arthralgias, and recurrent fever syndrome: a new autoinflammatory syndrome? J Rheumatol. 2019; 46(5).

92. Magadur-Joly G, Billaud E, Barrier JH, et al. Epidemiology of adult Still's disease: estimate of the incidence by a retrospective study in west France. Ann Rheum Dis. 1995; 54: 587-590.

93. Kurasawa M, Kotani K, Kurasawa G, et al. Adult-onset Still's disease in a patient over 80 years old successfully treated with low-dose methotrexate therapy. Age Ageing. 2007; 36(1): 104-106.

94. Wouters JM, van Rijswijk MH, van de Putte L, et al. Adult onset Still's disease in the elderly: a report of two cases. J Rheumatol.; 12(4): 791-3.

95. Yamaguchi M, Ohta A, Tsunematsu T, et al. Preliminary criteria for classification of adult Still's disease. J Rheumatol. 1992; 19(3): 424-430.

96. Lebrun D, MSDMea. Validation of the Fautrel classification criteria for adult-onset Still's disease. Semin Arthritis Rheum. 2018; 47(4): 578-585.

97. Rigante D, Vitale A, Natale MF, et al. A comprehensive comparison between pediatric and adult patients with periodic fever, aphthous stomatitis, pharyngitis, and cervical adenopathy (PFAPA) syndrome. J Clin Rheumatol. 2017; 36(2): 463-8.

98. Vitale A, Orlando I, Lopalco G, et al. Demographic, clinical and therapeutic findings in a monocentric cohort of adult patients with suspected PFAPA syndrome. Clin Exp Rheumatol. 2016; 34: 77-81.

99. Cattalini M, Soliani M, Rigante D, et al. Basic Characteristics of Adults with Periodic Fever, Aphthous Stomatitis, Pharyngitis, and Adenopathy Syndrome in Comparison with the Typical Pediatric Expression of Disease. Mediators Inflamm. 2015; 2015: 570418.

100. De Pauli S, Lega S, Pastore S, et al. Neither hereditary periodic fever nor periodic fever, aphthae, pharingitis, adenitis: Undifferentiated periodic fever in a tertiary pediatric center. World J Clin Pediatr. 2018; 7(1): 49-55.

101. Toplak N, Frenkel J, Ozen S, et al. An international registry on autoinflammatory diseases: the Eurofever experience. Ann Rheum Dis. 2012; 71(7): 1177-82. 
102. Georgin-Lavialle S, Stankovic K, Bachmeyer C, et al. Les maladies autoinflammatoires inclassées existent-t- elles? A propos de 56 cas adultes (abstract). Rev Med Interne. 2016; 37(1): A68.

103. Piram M, Frenkel J, Gattorno M, et al. A preliminary score for the assessment of disease activity in hereditary recurrent fevers: results from the AIDAI (Auto-Inflammatory Diseases Activity Index) Consensus Conference. Ann Rheum Dis. 2011; 70(2): 309-314.

104. Piram M, Koné-Paut I, Lachmann HJ, et al. Validation of the autoinflammatory diseases activity index (AIDAI) for hereditary recurrent fever syndromes. Ann Rheum Dis. 2014; 73(12): 2168-2173.

105. Vitale A, Rigante D, Lucherini OM, et al. Biological Treatments: New Weapons in the Management of Monogenic Autoinflammatory Disorders. Mediators Inflamm. 2013; 2013: 939847.

106. Georgin-Lavialle S, Rodrigues F, Hentgen V, et al. Panorama des maladies auto-inflammatoires. Rev Med Interne. 2018; 39: 214-232.

107. Sack, Jr GH. Serum amyloid A - a review. Mol Med. 2018; $24: 46$.

108. Berkun Y, Padeh S, Reichman B, et al. A single testing of serum amyloid A levels as a tool for diagnosis and treatment dilemmas in familial Mediterranean fever. Semin. Arthritis Rheum. Semin Arthritis Rheum. 2007; 37(3): 182-188.

109. Stankovic Stojanovica K, Hentgen V, Fellahi S, et al. Concordance between CRP and SAA in familial Mediterranean fever during attack-free period: A study of 218 patients. Clin Biochem. 2017; 50(4-5): 206-209.

110. Ben-Zvi I, Livneh A. Chronic inflammation in FMF: markers, risk factors, outcomes and therapy. Nat Rev Rheumatol. 2011; 7: 105-112.

111. Shinar Y, Obici L, Aksentijevich I, et al. Guidelines for the genetic diagnosis of hereditary recurrent fevers. Ann Rheum Dis. 2012;71(10):1599-1605. Ann Rheum Dis. 2012; 71(10): 1599-1605.

112. Federici S, Vanoni F, Ben-Chetrit E, et al. An International Delphi Survey for the Definition of New Classification Criteria for Familial Mediterranean Fever, Mevalonate Kinase Deficiency, TNF Receptor-associated Periodic Fever Syndromes, and Cryopyrin-associated Periodic Syndrome. J Rheumatol. 2019; 46(5).

113. Yao Q, Lacbawan F, Li J. Adult autoinflammatory disease frequency and our diagnostic experience in an adult autoinflammatory clinic. Semin Arthritis Rheum. 2016; 46(5): 633-637.

114. Omoyinmi E, Standing A, Keylock A, et al. Clinical impact of a targeted next-generation sequencing gene panel for autoinflammation and vasculitis. PLoS ONE. 2017; 12(7): e0181874.

115. Sarrabay G, Tachon G, Mechin D, et al. NGS for the diagnosis of autoinflammatory diseases: the experience of Montpellier. Pediatr Rheumatol. 2015; 13(1): O17.

116. Rowczenio DM, Lachmann HJ. How to prescribe a genetic test for the diagnosis of autoinflammatory diseases? Presse Med. 2019; 48(1): e49-e59.

117. Rusmini M, Federici S, Caroli F, et al. Next-generation sequencing and its initial applications for molecular diagnosis of systemic auto-inflammatory diseases. Ann Rheum Dis. 2016; 75: 1550-1557.
118. Ter Haar NM, Oswald M, Jeyaratnam J, et al. Recommendations for the management of autoinflammatory diseases. Ann Rheum Dis. 2015; 74: 1636-1644

119. Ter Haar N, Lachamnn HJ, Özen S, et al. Treatment of autoinflammatory diseases: results from the Eurofever Registry and a literature review. Ann Rheum Dis. 2013; 72(5): 678-85.

120. Kuemmerle-Deschner J, Schock A, Hansmann S, et al. THU0624 Colchicine: an effective treatment option for unclassified autoinflammatory diseases in children. Ann Rheum Dis. 2018; 77: 509.

121. Hausmann JS, Guben B, Anderson E, et al. Predicting Colchicine Response in Patients with Undefined Autoinflammatory Diseases [abstract]. 2017; 69(suppl 4).

122. Liantinioti G, Argyris AA, Protogerou AD, et al. The Role of Colchicine in the Treatment of Autoinflammatory Diseases. Curr Pharm Des. 2018; 24(6): 690-694.

123. Ozen S, Demirkaya E, Erer B, et al. EULAR recommendations for the management of familial Mediterranean fever. Ann Rheum Dis. 2016; 75: 644-651..

124. Hoffman HM. Therapy of autoinflammatory syndromes. J Allergy Clin Immunol. 2009; 124(6): 1129-1140.

125. Erbis G, Sergiichuk T, Bensele SM, et al. Determinants of health-related quality of life in children and adults with autoinflammatory diseases. Pediatr Rheumatol. 2015; 13(Suppl 1): P174.

126. Erbis G, Schmidt K, Hansmann S, et al. Living with autoinflammatory diseases: identifying unmet needs of children, adolescents and adults. Pediatr Rheumatol Online J. 2018; 16(1): 81.

127. Lepore L, Paloni G, Caorsi R, et al. Follow-up and quality of life of patients with cryopyrin-associated periodic syndromes treated with Anakinra. J Pediatr. 2010; 157(2): 310-315.

128. Duruoz MT, Unal C, Bingul DK, et al. Fatigue in familial Mediterranean fever and its relations with other clinical parameters. Rheumatol Int. 2018; 38(1): 75-81.

129. Lane T, Loeffler JM, Rowczenio DM, et al. Brief Report: AA Amyloidosis Complicating the Hereditary Periodic Fever Syndromes. Arthritis Rheum. 2013; 65: 1116-1121

130. Obici L, Merline G. Amyloidosis in autoinflammatory syndromes. Autoimmun Rev. 2012; 12(1): 14-17.

131. Lachmann HJ, Goodman HJ, Gilbertson JA, et al. Natural history and outcome in systemic AA amyloidosis. N Engl J Med. 2007; 356(23): 2361-71.

132. Ayar Y, Ersoy A, Oksuz MF, et al. Clinical outcomes and survival in AA amyloidosis

133. patients. Rev Bras Reumatol Engl Ed. 2017; 57(6): 535-544.

134. Ahbap E, Kara E, Sahutoglu T, et al. Outcome of 121 patients with renal amyloid a amyloidosis. 2014; 19(7): 644-649. 